\title{
Blockade of Lymphangiogenesis Shapes Tumor-Promoting Adipose Tissue Inflammation
}

Marek Wagner, Eli Sihn Steinskog, and Helge Wiig

From the Department of Biomedicine, University of Bergen, Bergen, Norway

Accepted for publication June 20, 2019.

Address correspondence to Marek Wagner, Ph.D., Department of Biomedicine, University of Bergen, Jonas Lies vei 91, 5009 Bergen, Norway. E-mail: marek.wagner@uib.no.

\begin{abstract}
Tumor-associated lymphangiogenesis correlates with lymph node metastasis and poor outcome in several human malignancies. In addition, the presence of functional lymphatic vessels regulates the formation of tumor inflammatory and immune microenvironments. Although lymphatic structures are often found deeply integrated into the fabric of adipose tissue, the impact of lymphangiogenesis on tumor-associated adipose tissue (AT) has not yet been investigated. Using K14-VEGFR3-Ig mice that constitutively express soluble vascular endothelial growth factor receptor (VEGFR) 3-Ig in the skin, scavenging VEGF-C and VEGF-D, the role of lymphangiogenesis in the generation of an inflammatory response within tumor-associated AT was studied. Macrophages expressing lymphatic vessel endothelial hyaluronan receptor-1 were found within peritumoral adipose tissue from melanoma-bearing K14-VEGFR3-Ig mice, which were further enriched with alternatively activated macrophages based on surface marker CD301/C-type lectin domain family 10 member A expression. The blockade of lymphangiogenesis also resulted in accumulation of the cytokine IL-6, which correlated with enhanced macrophage proliferation of the alternatively activated phenotype. Furthermore, melanomas co-implanted with freshly isolated adipose tissue macrophages grew more robustly than melanomas growing alone. In human cutaneous melanomas, adipocyte-selective FABP4 transcripts closely correlated with gene signatures of CLEC1OA and were associated with poor overall survival. These data suggest that the blockade of pathways regulating lymphatic vessel formation shapes an inflammatory response within tumor-associated AT by facilitating accumulation of tumor-promoting alternatively activated macrophages. (Am J Pathol 2019, 189: 2102-2114; https://doi.org/ 10.1016/j.ajpath.2019.06.010)
\end{abstract}

Tumor-associated lymphangiogenesis mediated by the lymphangiogenic factors vascular endothelial growth factor (VEGF)-C and VEGF-D through VEGF receptor (VEGFR)-3 activation contributes to malignant progression, at least in part, by facilitating metastatic spread to regional lymph nodes. ${ }^{1,2}$ However, lymphatic vessels and their drainage function also regulate local inflammation and antitumor host immune responses, thus serving multiple and intricate roles in tumor progression. ${ }^{1,3-5}$

Lymphatic structures such as collecting lymphatic vessels and lymph nodes are often found embedded in adipose tissue (AT). ${ }^{6}$ In addition to lipid-engorged adipocytes, AT harbors the stromal vascular fraction of cells, including preadipocytes, fibroblasts, vascular endothelial cells, and various immune cells with macrophages comprising the largest group. ${ }^{7,8}$ Evidence suggests that adipocytes together with classically activated macrophages secrete bioactive molecules, including cytokines that could be related to the development of obesity-associated low-grade systemic inflammation and insulin resistance. ${ }^{9-14}$ It has also been revealed that the blockage of VEGF-C and VEGF-D protects from insulin resistance and hepatic lipid accumulation through the accumulation of alternatively activated macrophages in subcutaneous AT from K14-VEGFR3-Ig mice that constitutively express soluble VEGFR3-Ig in the skin to prevent local VEGFR-3 signaling. ${ }^{15}$

Supported by Legat for Forskning av Kreftsykdommer ved Universitetet i Bergen grant 814172 (M.W.) and the Rakel og Otto Kr. Bruuns grant (H.W.).

Disclosures: None declared. 
Despite the fact that some tumors grow directly within AT or metastasize to the lymph nodes, AT is perhaps the most frequently overlooked stromal compartment in solid tumors. ${ }^{7}$ The progression of cutaneous melanomas, for example, is often noted when malignant cells enter a vertical growth phase and grow into subcutaneous tissue typically enriched with adipocytes and vascular endothelium. With the use of melanoma as a model, peritumoral AT was found to act as a proximate source of new blood vessels incorporated by the growing tumor mass and inflammatory cells, in particular macrophages. ${ }^{16,17}$ Whether lymphangiogenesis contributes to the generation of an inflammatory response within tumor-associated AT has not yet been assessed.

In this report, with the use of K14-VEGFR3-Ig mice, we unraveled the role of lymphangiogenesis in the generation of tumor-related inflammatory response within tumor-associated AT. ${ }^{15,18}$ In a melanoma model, AT juxtaposed to the growing tumors in K14-VEGFR3-Ig mice exhibited the presence of macrophages expressing the hyaluronan receptor lymphatic vessel endothelial hyaluronan receptor (LYVE)-1 and dense infiltration of alternatively activated macrophages based on surface marker CD301/C-type lectin domain family 10 member A (CLEC10A) expression. This observation was confirmed using mouse C3HBA adenocarcinoma cells implanted in the mammary fat pad of CHY mice, which carry a heterozygous inactivating mutation of VEGFR-3 resulting in an impaired dermal lymphangiogenesis. ${ }^{19}$ Furthermore, the blockade of lymphangiogenesis resulted in elevated levels of the cytokine IL-6 within tumorassociated AT from K14-VEGFR3-Ig mice and correlated with enhanced macrophage proliferation of the alternatively activated phenotype. Of importance, melanomas implanted in one of the subcutaneous AT depots of K14-VEGFR3-Ig mice together with freshly isolated AT macrophages grew faster, more robustly, and were more vascularized than those implanted alone. In human cutaneous melanomas, adipocyte-selective FABP4 transcripts closely correlated with CLEC1OA and were associated with poor overall survival.

These data suggest that the blockade of pathways regulating lymphatic vessel formation shapes tumor-promoting inflammation within tumor-associated AT by facilitating accumulation of alternatively activated macrophages.

\section{Materials and Methods}

Mice

Breeding, maintenance, and genotyping of K14-VEGFR3-Ig and CHY mice was performed as previously described. ${ }^{18,20}$ K14-VEGFR3-Ig male mice, a generous gift from Prof. Kari Alitalo (University of Helsinki, Finland), were on a C57BL/6 background and crossbred with wild-type (WT) C57Bl/6 female mice. Ten- to 11-week-old K14-VEGFR3Ig male mice and WT male littermates were used in experiments. Female $\mathrm{CHY}$ mice were on a $\mathrm{C} 3 \mathrm{H}$ background, and the breeding generated either $\mathrm{CHY}$ mice $\left(V E G F R 3^{+}\right.$/ Chy) or WT littermates $\left(V E G F R-3^{+/+}\right)$. Female mice of fertile age were used for C3HBA breast adenocarcinoma experiments. Animal experiments were performed according to the regulations of the Norwegian State Commission for Laboratory Animals that agreed with the European Convention for the Protection of Vertebrate Animals used for Experimental and Other Scientific Purposes and Council of Europe (ETS number 123). Experiments were conducted according to the approval from the Association for Assessment and Accreditation of Laboratory Animal Care International accredited Animal Care and Use Program at the University of Bergen (License number 7302 FOTS).

\section{Tumor Cells}

B16F10 murine melanoma cells were purchased from ATCC (Manassas, VA). C3HBA breast adenocarcinoma cells were obtained from the National Cancer Institute Division of Cancer Treatment and Diagnosis Tumor Repository (Frederick, MD). Tumor cells were incubated at $37^{\circ} \mathrm{C}$ in $5 \% \mathrm{CO}_{2}$ in Dulbecco's Modified Eagle's Medium (Sigma-Aldrich, St. Louis, MO), supplemented with $10 \%$ fetal bovine serum, nonessential amino acids, $100 \mathrm{U} / \mathrm{mL}$ penicillin, $100 \mu \mathrm{g} / \mathrm{mL}$ streptomycin, $4 \mathrm{mmol} / \mathrm{L}$ L-glutamine (all from Lonza, Basel, Switzerland), and plasmocin (InvivoGEN, San Diego, CA). Cells were detached and dissociated using trypsin-EDTA (Sigma-Aldrich) and passaged every other day.

\section{Implantation of Tumor Cells and Collection of Peritumoral AT}

For studies of tumor growth, B16F10 cells $\left(1 \times 10^{6}\right.$ cells $)$ were resuspended in $100 \mu \mathrm{L}$ of phosphate-buffered saline (PBS) and implanted in the anterior, subcutaneous AT depot or intradermally along the dorsal midline of syngeneic K14-VEGFR3-Ig mice or their WT littermates. C3HBA cells $\left(5 \times 10^{6}\right.$ cells $)$ were implanted in the abdominal mammary fat pad. For IL-6 neutralization, K14VEGFR3-Ig mice were administered daily an anti-IL-6 $(0.5 \mu \mathrm{g}$ in $100 \mu \mathrm{L}$ of PBS) antibody (BioXCell, West Lebanon, NH) or PBS intraperitoneally. Tumor size was measured daily with the use of a vernier caliper. Tumor volume was measured with calipers and calculated with the formula $a^{2} b \times(\pi / 6)$, where $a$ and $b$ stand for the shorter and longer diameter of the tumors, respectively. Animals were euthanized by $\mathrm{CO}_{2}$ inhalation, and tumor samples were collected. For AT studies, peritumoral AT was microdissected away from the tumor mass with the use of a stereoscopic microscope. Control AT from the counterpart depot was collected from age- and sex-matched animals. Tumor and AT samples were weighed and then fixed in $4 \%$ paraformaldehyde, embedded in paraffin, and cut into $4-\mu \mathrm{m}$ thick sections. 


\section{Morphometric Analysis of Adipocytes}

Morphometric analysis of adipocytes $(n>250)$ from AT samples from three different animals per group was performed with NIS-elements AR software version 4.11 (Nikon, Tokyo, Japan).

\section{Immunohistochemistry and Immunofluorescence}

For immunohistochemical staining, tissue sections were deparaffinized and rehydrated before antigen retrieval at $98^{\circ} \mathrm{C}$ in water bath for 20 to 60 minutes in $0.01 \mathrm{~mol} / \mathrm{L}$ citrate buffer (pH 6.0). For F4/80 staining, treatment with Proteinase K (Dako, Carpinteria, CA) was used according to the manufacturer's instructions provided. After being blocked with diluted serum from the secondary antibody host for 30 minutes, the sections were incubated with the primary antibody overnight at $4^{\circ} \mathrm{C}$. Blocking of endogenous peroxidase was performed with $3 \% \mathrm{H}_{2} \mathrm{O}_{2}$ (Sigma-Aldrich) for 20 minutes. Subsequently, the sections were incubated with a biotinylated anti-rat, anti-rabbit, or anti-goat secondary antibody (Vector Laboratories, Burlingame, CA) for 45 minutes at room temperature. Avidin-biotinperoxidase was used to augment the antigen-antibody complex reaction for 45 minutes according to the manufacturer's instructions provided (Vectastain ABC Kit; Vector Laboratories) and stained for 1 to 10 minutes with diaminobenzidine tetrahydrochloride (Vector Laboratories). The sections were counterstained with hematoxylin (Thermo Fisher Scientific, Waltham, MA), dehydrated, and mounted with the use of Entellan (Merck Millipore, Darmstadt, Germany). To ensure the specificity of the immunoreactions, parallel sections were run for all of the experiments with the primary antibody omitted. The following primary antibodies were used: rabbit anti-Perilipin-1 (dilution 1:200; Cell Signaling Technology, Danvers, MA), rabbit anti-LYVE1 (dilution 1:100; Abcam, Cambridge, UK), rat anti-F4/80 (dilution 1:50; Abcam), rat antiCD301 (dilution 1:50; Bio-Rad AbD Serotec, Puchheim, Germany), rabbit anti-IL-4 receptor (IL-4R; dilution 1:250; Abcam), and goat anti-CD31 (dilution 1:100; Santa Cruz Biotechnology, Dallas, TX). For immunofluorescence staining, the sections were incubated with the primary antibody overnight at $4^{\circ} \mathrm{C}$. A mixture of the corresponding secondary antibodies was applied for 45 minutes at room temperature thereafter. Finally, the slides were mounted with the use of DAPI mounting medium (Vector Laboratories). The following primary antibodies were used: rabbit anti-LYVE1 (dilution 1:100; Abcam), rat anti-LYVE1 (dilution 1:100; R\&D Systems, Minneapolis, $\mathrm{MN}$ ), rat anti-F4/80 (dilution 1:50; Abcam), rabbit anti-CD11b (dilution 1:200; Abcam), rat anti-CD301 (dilution 1:50; Bio$\mathrm{Rad}$ AbD Serotec), rabbit anti-IL-4R (dilution 1:250; Abcam), rabbit anti-Ki67 (dilution 1:100; Abcam), and rat antibromodeoxyuridine [BrdU; BU1/75(ICR1); dilution 1:100; Bio-Rad AbD Serotec]. The secondary antibodies used were: cyanine 2 anti-rat IgG (dilution 1:200; Jackson ImmunoResearch, West Grove, PA), cyanine 3 anti-rabbit IgG (dilution 1:200; Jackson ImmunoResearch), Alexa Fluor 488 anti-rat IgG
$(\mathrm{H}+\mathrm{L})$ (dilution 1:200; Thermo Fisher Scientific), and Alexa Fluor 568 anti-rabbit $\operatorname{IgG}(\mathrm{H}+\mathrm{L})$ (dilution 1:200; Thermo Fisher Scientific).

\section{In Vivo BrdU Incorporation}

Mice were intraperitoneally administered $200 \mu \mathrm{L}$ of BrdU solution $(10 \mathrm{mg} / \mathrm{mL})$ (Sigma-Aldrich). Incorporation of BrdU into proliferating cells within subcutaneous AT was analyzed 4 hours after injection by immunofluorescence staining.

\section{ELISA}

Mouse IL-6 enzyme-linked immunosorbent assay (ELISA) kit was purchased from Sigma-Aldrich. ELISA was performed as recommended by the manufacturer's instructions provided. For the assessment of IL-6, whole AT protein lysates were used. Briefly, peritumoral and control AT samples were homogenized, and cells were sonicated in a total protein lysis buffer $(50 \mathrm{mmol} / \mathrm{L}$ TRIS-HCl, $\mathrm{pH} 7.5,150 \mathrm{mmol} / \mathrm{L}$ $\mathrm{NaCl}, 0.1 \%$ SDS, $1 \%$ deoxycholate, $1 \%$ Triton X-100) supplemented with a protease and phosphatase inhibitors (Roche, Basel, Switzerland). Measurements were done in duplicate.

\section{FACS}

Freshly collected subcutaneous AT samples pooled from 10 naive C57BL/6 mice were digested with a mixture of collagenase Type-2 (1 mg/mL; Worthington, Lakewood, NJ), dispase II $(2.5 \mu \mathrm{g} / \mathrm{mL}$; Roche $)$, and DNase $(1 \mathrm{mg} / \mathrm{mL}$; Worthington) for 1 hour at $37^{\circ} \mathrm{C}$ with continuous rotation. The dissociated tissue was centrifuged at $186 \times g$ for 10 minutes. To remove debris, the pelleted stromal vascular fraction was filtered through 70- $\mu \mathrm{m}$ mesh cell strainer (BD Bioscience, San Jose, CA). Contaminating erythrocytes were lyzed using red blood cell lysis buffer (BD Bioscience). Cells were resuspended in fluorescence-activated cell sorting (FACS) buffer $(0.5 \% \mathrm{w} / \mathrm{v}$ bovine serum albumin, $0.5 \mathrm{~mol} / \mathrm{L}$ EDTA in sterile PBS) and blocked with FcR-blocking reagent (Miltenyi Biotec, Bergisch Gladbach, Germany), to prevent nonspecific binding. After incubation for 30 minutes at $4{ }^{\circ} \mathrm{C}$ with appropriate antibodies, cells were sorted with the use of BD FACSAria Cell Sorting System with BD FACSDiva software version 8.0 (BD Bioscience). The following antibodies were used: rat anti-CD11b:RPE (Bio-Rad AbD Serotec) and rat anti-F4/80:AF488 (Bio-Rad AbD Serotec). For tumor growth studies, B16F10 cells $\left(2.5 \times 10^{5}\right.$ cells $)$ alone or admixed with $\mathrm{CD} 11 \mathrm{~b}^{+} \mathrm{F} 4 / 80^{+}$cells $\left(2.5 \times 10^{5}\right.$ cells $)$ were injected into the contralateral anterior, subcutaneous AT depots of syngeneic K14-VEGFR3-Ig mice of both sexes.

\section{Database Analysis}

The association between expression levels of FABP4 and survival rates of 458 melanoma patients from the publicly available The Cancer Genome Atlas (TCGA; http:// 
cancergenome.nih.gov, last accessed March 12, 2019) database was determined with the Oncolnc platform ( $w w w$. oncolnc.org, last accessed March 12, 2019). According to the expression levels of $F A B P 4$, melanoma samples $(n=458)$ were assigned into two groups of low $(n=229)$ and high $(n=229)$ expression levels of FABP4. Expression data were extracted and the Kaplan-Meier estimation curve was plotted with Prism software version 6. 0 (GraphPad Software Inc., San Diego, CA).

The gene expression correlation analysis was performed on the Tumour Skin Cutaneous Melanoma - TCGA - 470 rsem - tcgars data set that contained data from 470 melanoma patients retrieved from the publicly available TCGA (http://cancergenome.nih.gov) database with the use of the microarray analysis and visualization platform R2 ( $h t t p: / /$ r2.amc.nl, last accessed February 13, 2019). Pearson's correlation coefficient, $r$, was calculated with the transform $2 \log$ setting. Expression data of the genes of interest were extracted and plotted with Prism software (GraphPad Software Inc.).

\section{Statistical Analysis}

The statistical significance was assessed by unpaired $t$-test or one-way analysis of variance followed by Tukey's multiple comparisons test, using Prism software (GraphPad Software
A
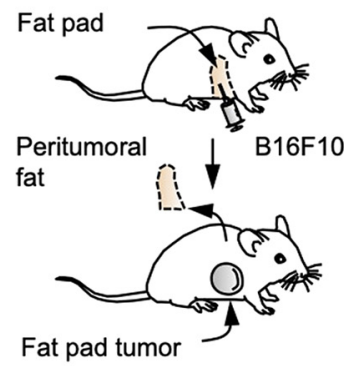

B

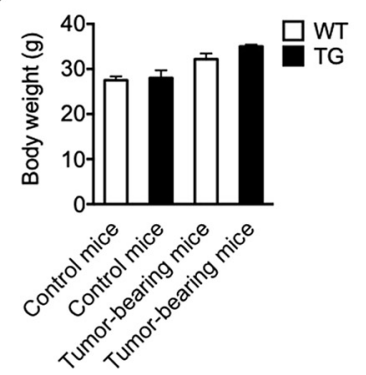

C

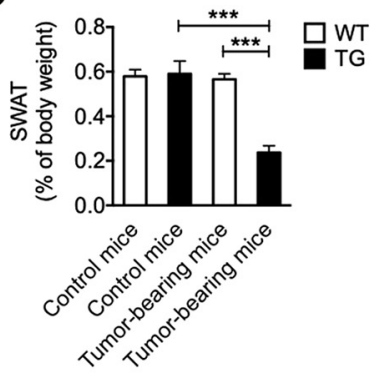

D

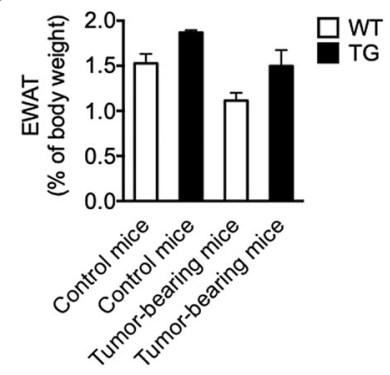

E

빺

G
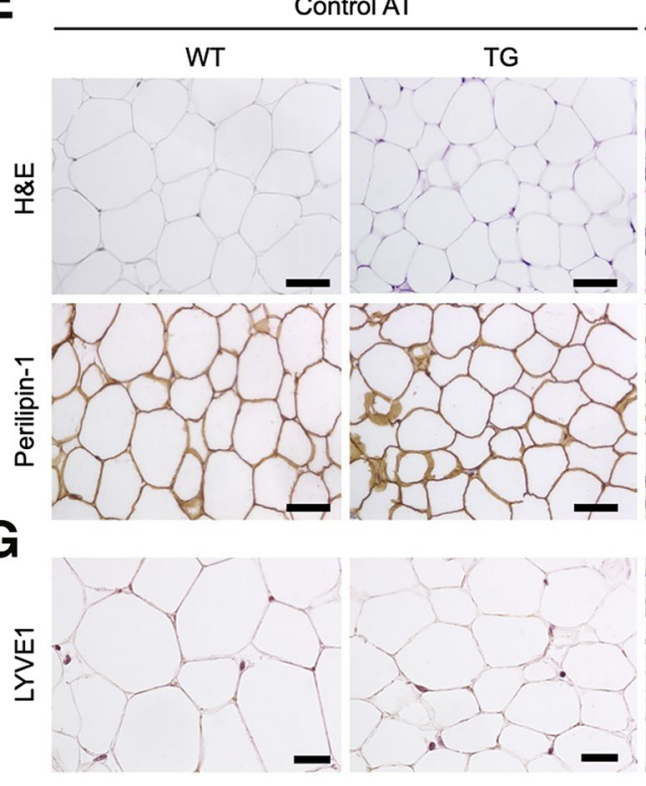

Peritumoral AT

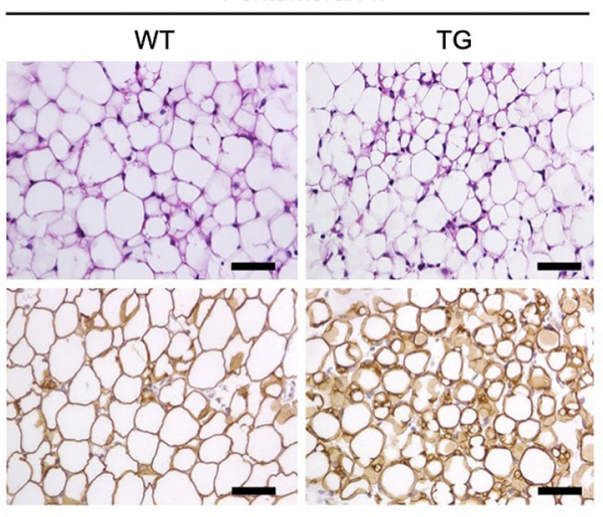

$\mathbf{F}$

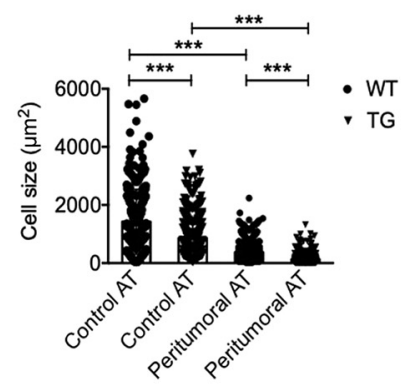

$\mathrm{H}$

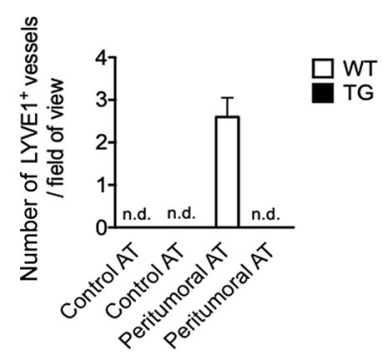

Figure 1 Tumor-bearing K14-VEGFR3-Ig mice demonstrate decreased peritumoral adipose tissue (AT) mass and reduced adipocyte size. A: Graphical representation of the study design. B16F10 melanoma cells $\left(1 \times 10^{6}\right.$ cells) were implanted in the fat pad of wild-type (WT) or K14-VEGFR3-Ig transgenic (TG) mice. B: Body weights of control and tumor-bearing mice of two genotypes. C and D: Weights of subcutaneous white AT (SWAT; C) and epididymal white AT (EWAT; D) from control and tumor-bearing mice of two genotypes. Decreased tissue mass reveals atrophy of peritumoral AT from TG mice. E: Hematoxylin-eosin (H\&E) histologic examination of control and peritumoral AT from WT and TG mice. Immunohistochemical analysis of control and peritumoral AT from WT and TG mice using an antibody against the Perilipin-1, an adipocyte-specific lipid-coated protein. F: Adipocyte size quantification demonstrates drastically reduced size of fat cells from peritumoral AT from TG mice. G: Immunohistochemical analysis of control and peritumoral AT from WT and TG mice using an antibody against the lymphatic vessel endothelial hyaluronan receptor (LYVE) 1, a lymphatic endothelium-specific hyaluronan receptor. Arrows mark LYVE1 ${ }^{+}$cells. Asterisk marks LYVE1 ${ }^{+}$vessel. H: Quantification of lymphatic vessels reveals presence of LYVE1 ${ }^{+}$structures in peritumoral AT from WT mice only. Data are expressed as means \pm SEM. $n=3$ to 6 mice per group (B-D); $n>250$ cells per group $(\mathbf{F}) ; n=3$ to 4 mice per group (H). ${ }^{* * *} P<0.001$ (statistical analysis with one-way analysis of variance followed by Tukey's multiple comparisons test). Scale bars: $50 \mu \mathrm{m}$ (E); $25 \mu \mathrm{m}$ (G). n.d., not detected. 
A
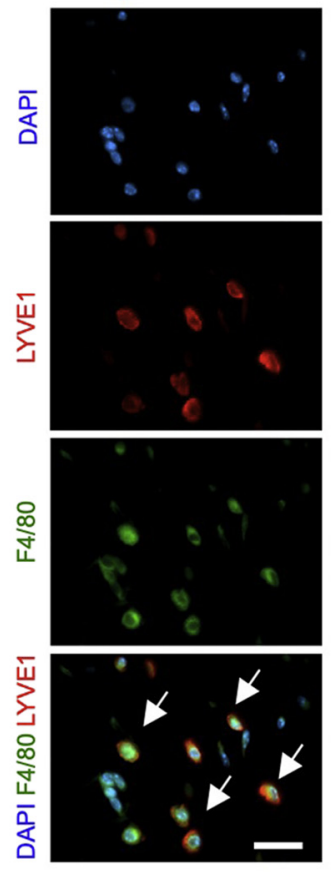

B
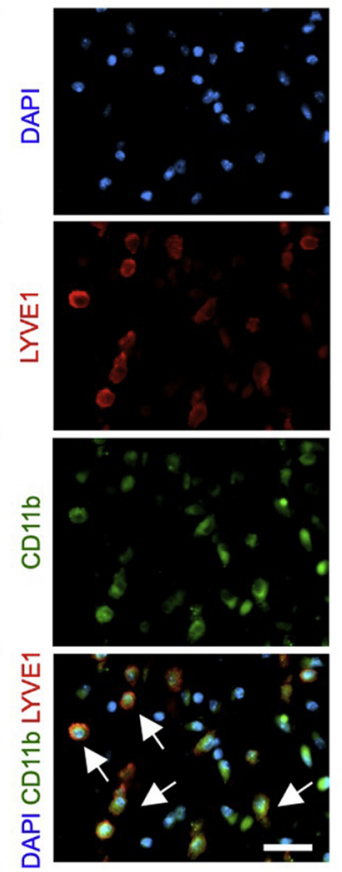

C

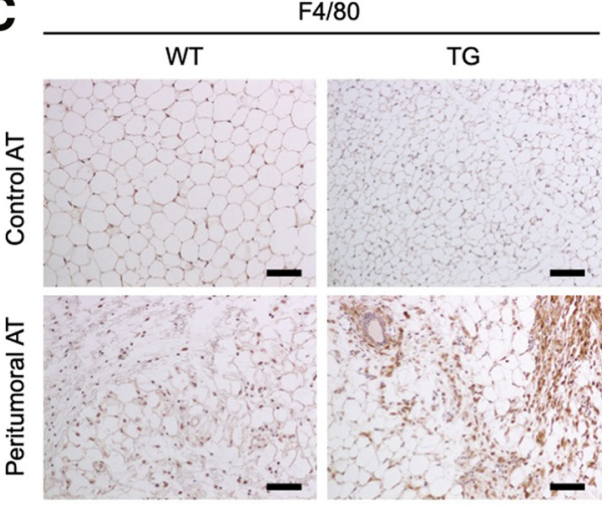

E

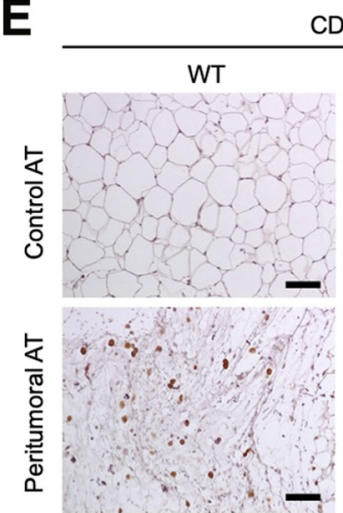

D

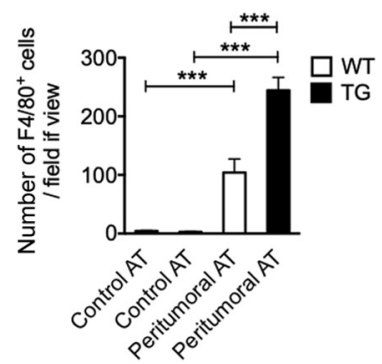

$\mathbf{F}$

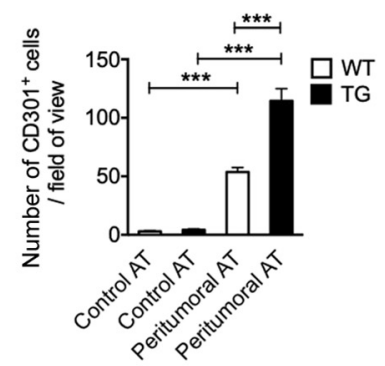

Figure 2 Peritumoral adipose tissue (AT) from K14-VEGFR3-Ig mice shows increased accumulation of macrophages of alternatively activated phenotype based on surface marker CD301 expression. A and B: Immunofluorescence analysis reveals lymphatic vessel endothelial hyaluronan receptor (LYVE) $1^{+}$cells coexpressing murine macrophage markers F4/80 (A) and Cd11b (B). Arrows mark LYVE1 ${ }^{+}$macrophages. C: Immunohistochemical analysis of control and peritumoral AT from wild-type (WT) and transgenic (TG) mice using an antibody against the F4/80. D: Quantification of F4/80+ cells demonstrates increased infiltration of macrophages in peritumoral AT from TG mice. E: Immunohistochemical analysis of control and peritumoral AT from WT and TG mice using an antibody against the CD301, a marker of alternatively activated macrophages. F: Quantification of $\operatorname{CD} 301^{+}$cells reveals increased accumulation of alternatively activated macrophages in peritumoral AT from TG mice. Data are expressed as means \pm SEM. $n=3$ to 4 mice per group (D and $\mathbf{F})$. ${ }^{* * *} P<0.001$ (statistical analysis with one-way analysis of variance followed by Tukey's multiple comparisons test). Scale bars: $25 \mu \mathrm{m}$ (A and B); $50 \mu \mathrm{m}$ (C and E).

Inc.). The value of $P<0.05$ was considered statistically significant. Whenever possible, the investigators (M.W. and E.S.S.) were partially blinded for assessing the outcome (eg, immunohistochemistry). To determine the correlation between gene expression levels in skin cutaneous melanoma patients in the TCGA data set, Pearson's correlation coefficient, $r$, was calculated. To demonstrate the association between gene expression levels and survival rate of skin cutaneous melanoma patients in the TCGA data set, multivariate Cox regression and Kaplan-Meier analysis were performed.

\section{Results}

Peritumoral AT from K14-VEGFR3-Ig Mice Is Atrophied and Typified by Decreased Adipocyte Size

In mice there are two subcutaneous white AT depots (or fat pads) located along the scapulae (descending from the neck to the axillae). We took advantage of their anatomic location and implanted B16F10 murine melanoma cells $\left(1 \times 10^{6}\right.$ cells) directly in one of the fat pads of K14-VEGFR3-Ig mice (referred to as TG in the figures) or WT littermates, to characterize peritumoral AT (Figure 1A). At 14 days after implantation, peritumoral AT was microdissected away from the surrounding tumor mass and compared with control AT. Control and tumor-bearing mice had a comparable body weight for both genotypes (Figure 1B). Although the weight of control AT was similar between WT and K14-VEGFR3-Ig mice, the weight of peritumoral AT from transgenic mice was on average twofold lower than that from WT littermates (Figure 1C). The weight of epididymal white AT between control and tumor-bearing mice for both genotypes was not significantly changed (Figure 1D). Under microscopic examination of hematoxylin and eosin-stained sections, adipocytes from peritumoral AT for both genotypes appeared smaller than their normal counterparts (Figure 1E). This observation was confirmed by staining AT sections with an antibody against the lipid dropletassociated protein, perilipin-1 (Figure 1E). Furthermore, when measured with the use of NIS-elements AR software, adipocytes from peritumoral AT from K14-VEGFR3-Ig mice were approximately $60 \%$ smaller than adipocytes from WT mice (Figure 1F). Decreased adipocyte size from control AT from K14-VEGFR3-Ig mice was also observed 
A

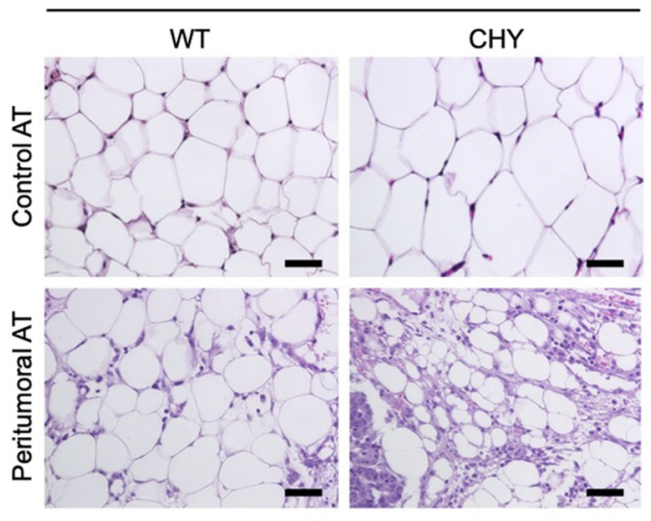

C

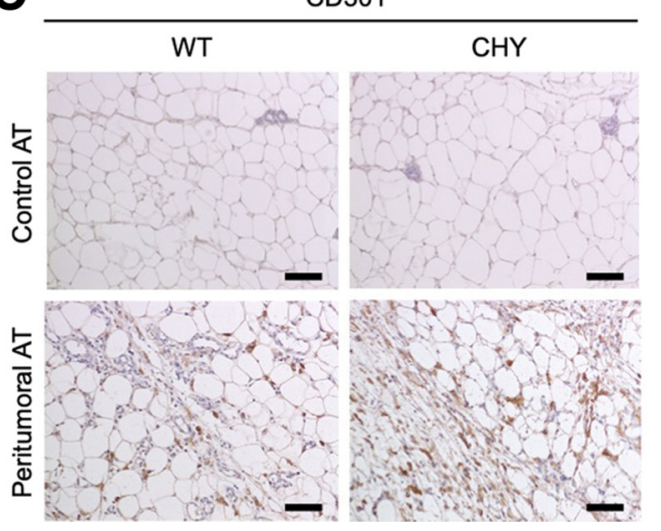

B

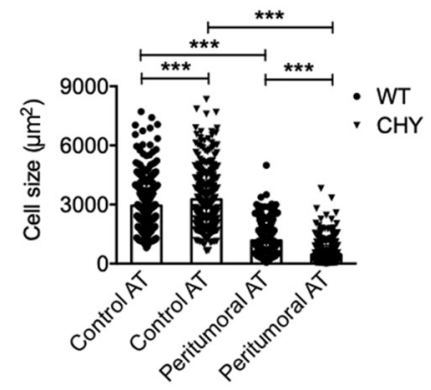

D

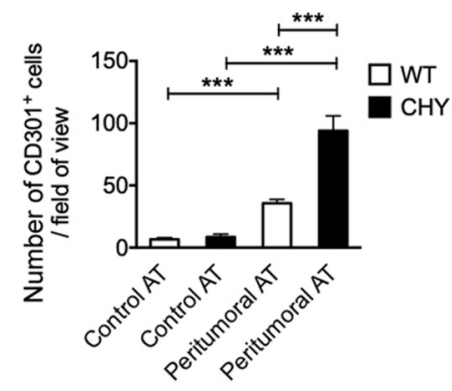

Figure 3 Peritumoral adipose tissue (AT) from CHY mice shows reduced adipocyte size and increased accumulation of macrophages of alternatively activated phenotype based on surface marker CD301 expression. A and B: Hematoxylineosin (H\&E) histologic examination (A) and quantification (B) of adipocyte size from control and peritumoral AT from wild-type (WT) and CHY mice. C and D: Immunohistochemical analysis of control and peritumoral AT from WT and CHY mice using an antibody against the CD301 (C) and quantification of $\mathrm{CD} 01^{+}$cells (D). Data are expressed as means \pm SEM. $n>300$ cells per group (A and $\mathbf{B}$ ); $n=3$ mice per group (C and $\mathbf{D})$. $* * * P<0.001$ (statistical analysis with one-way analysis of variance followed by Tukey's multiple comparisons test). Scale bars: $50 \mu \mathrm{m}$ (A and $\mathbf{C})$. compared with WT littermates. The mean sizes were $1421 \pm 67 \mu^{2}$ and $865 \pm 34 \mu^{2}$ for adipocytes from control AT from WT and K14-VEGFR3-Ig mice, respectively ( $n>250$ cells per group). For adipocytes from peritumoral AT the mean sizes were $371 \pm 11 \mu^{2}$ for WT mice and $129 \pm 4 \mu \mathrm{m}^{2}$ for transgenic mice $(n>250$ cells per group) (Figure 1F). Similarly, the number of adipocytes was reduced in peritumoral AT from transgenic mice compared with WT littermates (data not shown).

The lymphatic vasculature in the AT from K14-VEGFR3Ig and WT mice was next investigated (Figure $1 \mathrm{G}$ ). Immunohistochemical analysis of control and peritumoral AT revealed the existence of lymphatic vessels within peritumoral AT from WT mice only as judged by the presence of $\mathrm{LYVE}^{+}{ }^{+}$structures (Figure 1H). However, peritumoral AT from K14-VEGFR3-Ig mice was infiltrated by what appeared to be a population of variably shaped LYVE1 ${ }^{+}$single cells rather than lymphatic endothelial cells forming tube-like structures (Figure 1G).

\section{Peritumoral AT from K14-VEGFR3-Ig Mice Is Characterized by Local Proliferation of Alternatively Polarized Macrophages}

Because macrophages are known to express LYVE1, the colocalization of LYVE1 was examined with a murine macrophage and myeloid cell markers, F4/80 and CD11b, respectively. Indeed, immunofluorescence analysis revealed the expression of LYVE1 by $\mathrm{F} 4 / 80^{+}$and $\mathrm{CD}^{+} 1 \mathrm{~b}^{+}$cells (Figure 2, A and B). Next, macrophages in control and peritumoral AT were quantitatively and qualitatively characterized from both genotypes. ${ }^{21-24}$ Immunohistochemical analysis revealed abundance of $\mathrm{F} 4 / 80^{+}$macrophages in peritumoral versus control AT with a significant increase observed in K14-VEGFR3-Ig mice compared with WT mice (Figure 2, C and D). Further analysis of the peritumoral AT from transgenic mice demonstrated an increased number of macrophages expressing the C-type lectin 10A (CLEC10A/CD301), a marker of alternative polarization compared with peritumoral AT from WT littermates (Figure 2, E and F). No significant difference was observed in the number of $\mathrm{CD}^{+} \mathrm{T}$ cells in peritumoral AT from transgenic versus WT mice (data not shown).

The observed phenotype was confirmed with another model of lymphatic insufficiency, CHY mice, which carry a heterozygous inactivating mutation of VEGFR-3. Syngeneic C3HBA adenocarcinoma cells were implanted in the mammary fat pad of CHY mice and WT littermates. At 16 days after implantation, adipocytes were found from peritumoral AT from CHY mice approximately 60\% smaller compared with WT mice under microscopic examination of hematoxylin and eosin-stained sections (Figure 3A). In contrast, the size of adipocytes from control AT from $\mathrm{CHY}$ mice was found to be significantly increased compared with 
A

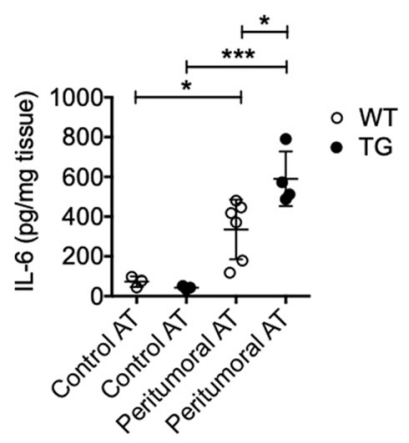

B

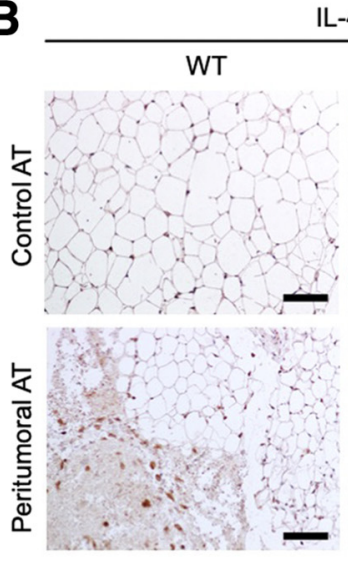

IL-4R

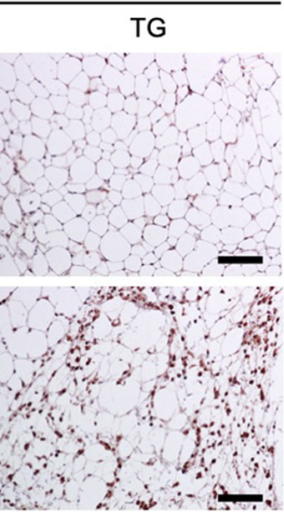

C

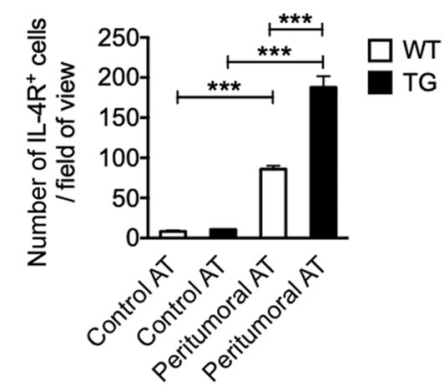

$\mathbf{F}$

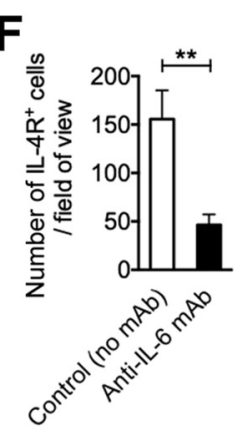

H

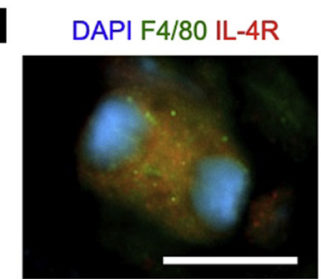

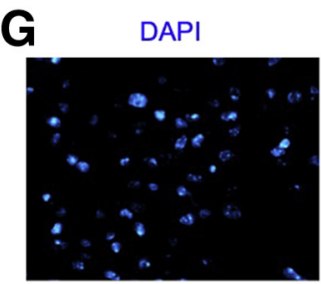
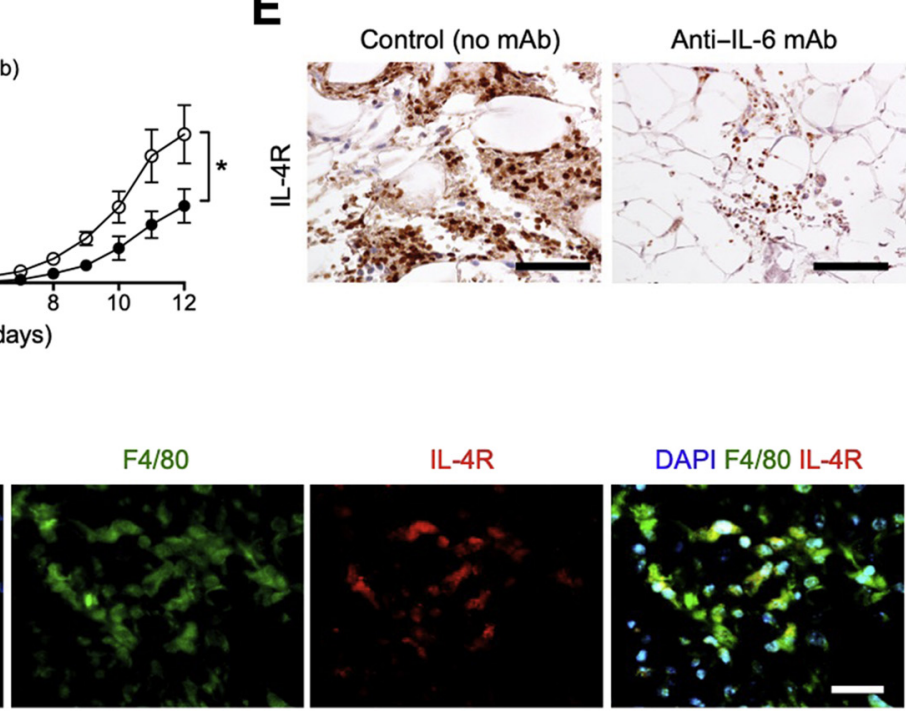

Figure 4 Increased levels of IL-6 characterize peritumoral adipose tissue (AT) from K14-VEGFR3-Ig mice. A: IL-6 levels in control and peritumoral AT from wild-type (WT) and transgenic (TG) mice measured by enzyme-linked immunosorbent assay. B: Immunohistochemical analysis of control and peritumoral AT from WT and TG mice using an antibody against the IL-4 receptor. C: Quantification reveals increased accumulation of IL-4R ${ }^{+}$cells in peritumoral AT from TG mice. D: Tumor growth profiles in TG mice treated every day with anti-IL-6 monoclonal antibody [mAb; $0.5 \mu \mathrm{g}$ in $100 \mu \mathrm{L}$ of phosphate-buffered saline (PBS)] or PBS (Control). E and F: Immunohistochemical analysis of peritumoral AT from TG mice using an antibody against the IL-4 receptor (IL-4R; E) and quantification of IL-4R ${ }^{+}$cells (F). G: Immunofluorescence analysis reveals $\mathrm{F} 4 / 80^{+}$macrophages coexpressing IL-4 receptor. H: The mitotic figures in $\mathrm{F} 4 / 80^{+} \mathrm{IL}-$ $4 \mathrm{R}^{+}$macrophages are occasionally observed in peritumoral AT from TG mice. Data are expressed as means \pm SEM. $n=4$ to 6 mice per group (A); $n=3$ mice per group (C); $n=5$ to 6 mice per group (D); $n=4$ mice per group $(\mathbf{E}$ and $\mathbf{F})$. ${ }^{*} P<0.05,{ }^{*} P<0.01$, and ${ }^{* * *} P<0.001$ (statistical analysis with one-way analysis of variance followed by Tukey's multiple comparisons test). Scale bars: $100 \mu \mathrm{m}$ (B); $50 \mu \mathrm{m}(\mathbf{E}) ; 25 \mu \mathrm{m}(\mathbf{G}) ; 10 \mu \mathrm{m}(\mathbf{H})$.

WT counterparts (Figure 3A). The mean sizes were $2934 \pm 69 \mu \mathrm{m}^{2}$ and $3258 \pm 73 \mu^{2}$ for adipocytes from control AT from WT and CHY mice, respectively $(n>300$ cells per group) (Figure 3B). For adipocytes from peritumoral AT the mean sizes were $1174 \pm 44 \mu \mathrm{m}^{2}$ for WT mice and $457 \pm 19 \mu \mathrm{m}^{2}$ for mutated mice $(n>300$ cells per group) (Figure 3B). In addition, immunohistochemical analysis revealed a 2.5 -fold increase in the number of macrophages expressing CD301 in peritumoral AT from mutated mice (Figure 3, C and D). No difference was observed in the number of $\mathrm{CD} 301^{+}$macrophages in control AT from mutated versus WT mice (Figure 3, C and D).

The expression of IL-6, compared with control AT, was strikingly up-regulated in peritumoral AT from C57BL/6 mice. ${ }^{16}$ In addition, it was recently revealed that IL- 6 signaling promoted alternative macrophage polarization through the induction of IL-4R expression. $^{25-27}$ Thus, it was speculated that peritumoral AT-derived IL-6 might regulate macrophage polarization in this model. It was first confirmed that IL-6 was expressed in peritumoral AT from both WT and K14VEGFR3-Ig mice. IL-6 expression was approximately 1.7fold higher in peritumoral AT from K14-VEGFR3-Ig mice than from WT littermates (Figure 4A). Next, an increase abundance of cells expressing IL-4R was found in peritumoral AT from K14-VEGFR3-Ig mice as revealed by immunohistochemical analysis (Figure 4, B and C). Of note, neutralization of IL-6 by in vivo treatment with anti-IL-6 antibody significantly inhibited the growth of melanomas (Figure 4D) and decreased the number of $\mathrm{IL}_{-} 4 \mathrm{R}^{+}$cells within the peritumoral AT from K14-VEGFR3-Ig mice compared with controls (Figure 4, E 
A
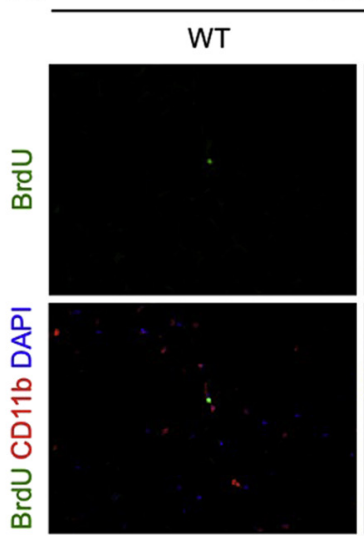

C

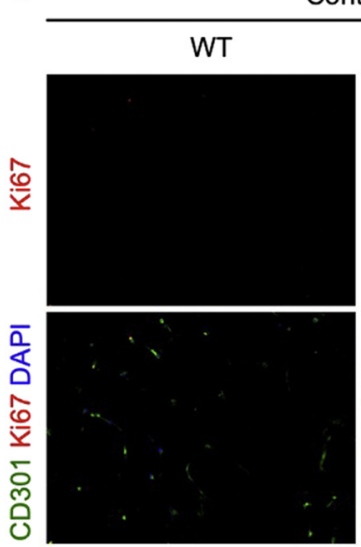

Control AT
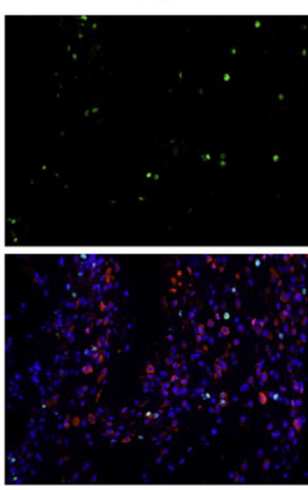

Control AT

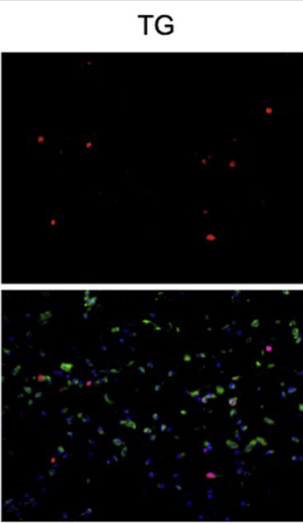

Peritumoral AT WT
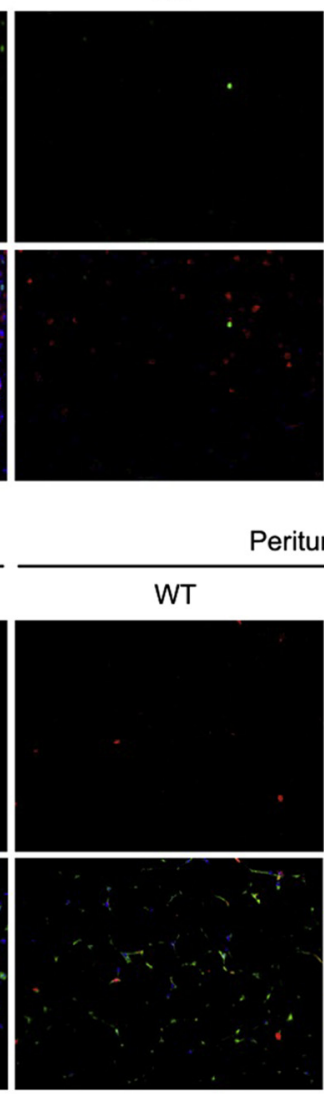

Peritumoral AT

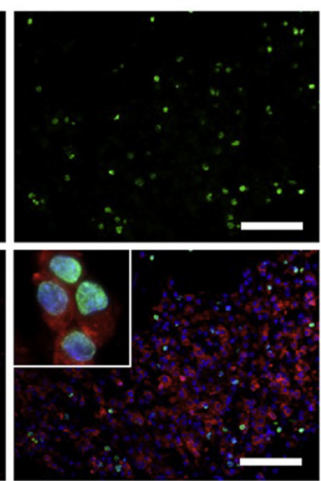

\section{D}

B
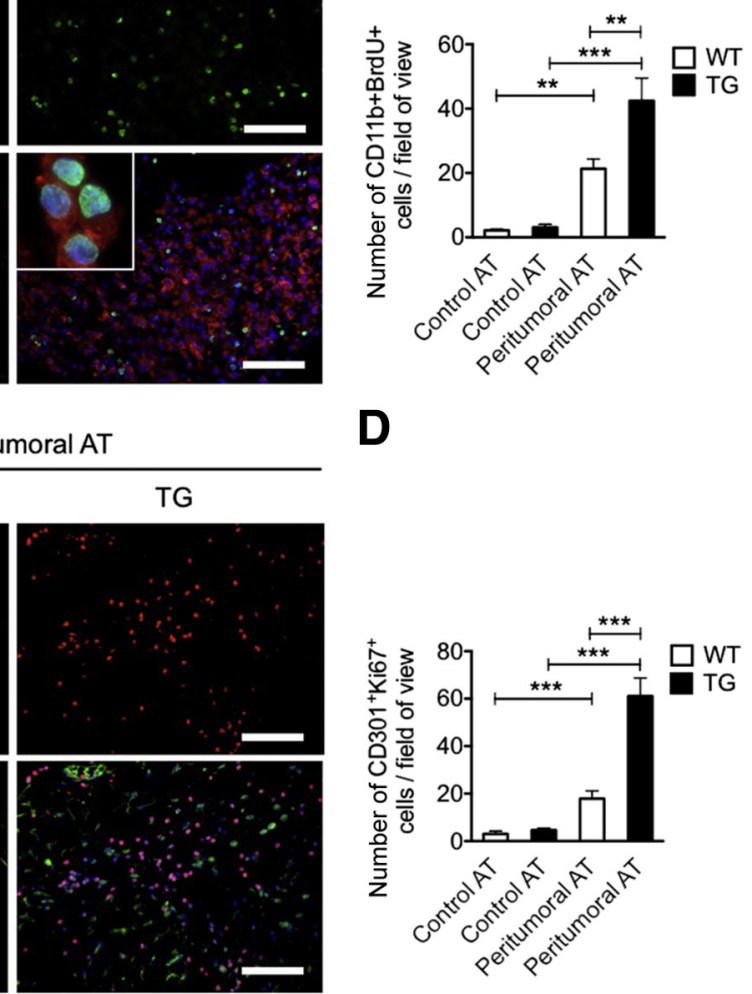

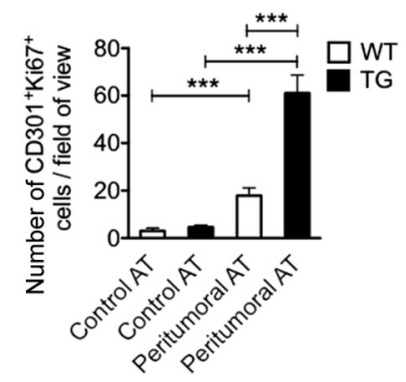

Figure 5 Peritumoral adipose tissue (AT) from transgenic (TG) mice demonstrates increased accumulation of proliferating macrophages. A and B: Immunofluorescence analysis (A) and quantification (B) of bromodeoxyuridine (BrdU) ${ }^{+} \mathrm{CD} 11 \mathrm{~b}^{+}$cells in control and peritumoral AT from wild-type (WT) and TG mice. The

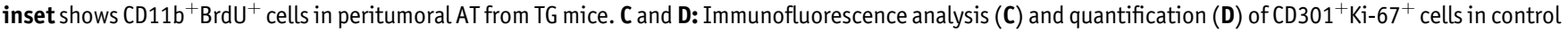

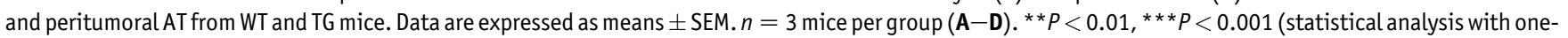
way analysis of variance followed by Tukey's multiple comparisons test). Scale bars: $100 \mu \mathrm{m}$ (A, main image, and $\mathbf{C})$. Original magnification, $\times 63$ (A, inset).

and F). Finally, the colocalization of IL-4R with F4/80 confirmed IL-4R expression by macrophages (Figure 4G). On occasion, mitotic figures were also detected within peritumoral AT from K14-VEGFR3-Ig mice (Figure 4H).

To better assess the extent of macrophage proliferation in peritumoral AT, cell proliferation was analyzed in vivo by administrating BrdU. Incorporation of BrdU was measured by cells within AT 4 hours after BrdU injection. Almost twice as many $\mathrm{BrdU}^{+} \mathrm{CD} 11 \mathrm{~b}^{+}$cells were detected in peritumoral AT from K14-VEGFR3-Ig mice compared with WT littermates (Figure 5A and B). Peritumoral AT was also found from K14-VEGFR3-Ig mice highly infiltrated with $\mathrm{Ki}-67^{+}$macrophages expressing CD301, indicative of their alternative form of activation (Figure 5, C and D). ${ }^{28}$

\section{Macrophages within Peritumoral AT Increase Tumor Growth and Vascularization}

To assess tumor growth and vascularization, B16F10 murine melanoma cells $\left(1 \times 10^{6}\right.$ cells $)$ were directly implanted in one of the fat pads or in the dorsal dermis (far from the fat pads) of K14-VEGFR3-Ig or WT mice (Figure 6A). Melanomas implanted in the fat pad of K14-VEGFR3-Ig mice grew faster and more robustly than with WT littermates (Figure 6B). The lack of dermal lymphatic vessels, however, did not affect the growth of tumors at a site distant from the AT depots (Figure 6B). At 14 days after implantation, tumors growing in the fat pads weighed almost twice and thrice as much as melanomas implanted in the dorsal dermis in K14-VEGFR3-Ig $(2.5 \pm 0.3 \mathrm{~g}$ versus $0.7 \pm 0.1 \mathrm{~g} ; n=6$; $P<0.001)$ and WT $(1.7 \pm 0.2 \mathrm{~g}$ versus $0.8 \pm 0.2 \mathrm{~g} ; n=6$; $P<0.05$ ) mice, respectively (Figure 6C). Immunohistochemical staining revealed increased blood vessel density, as analyzed by the numbers of $\mathrm{CD} 31^{+}$structures per area, within tumors growing in AT in both K14-VEGFR3-Ig and WT mice (Figure 6, D and E). Of importance, however, melanomas implanted in the fat pad of K14-VEGFR3-Ig mice were significantly more vascularized than with WT littermates (Figure 6, D and E).

It was therefore speculated that the increased growth of melanomas implanted in K14-VEGFR3-Ig mice was related to an elevated number of macrophages within 
A

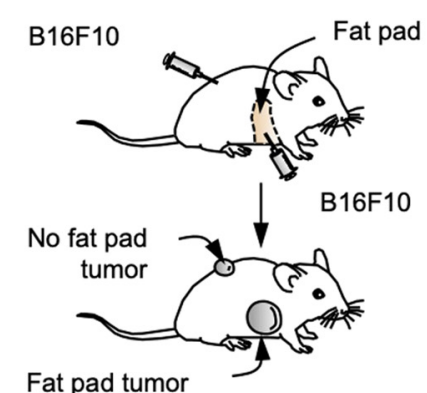

Fat pad tumor
B

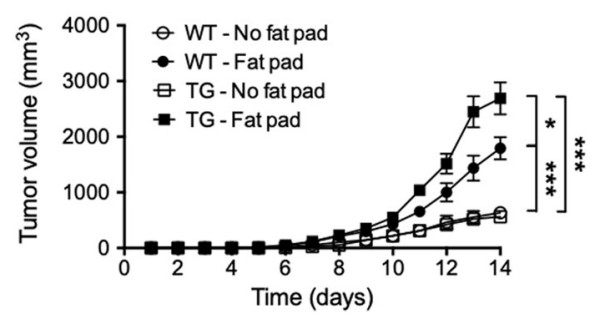

C

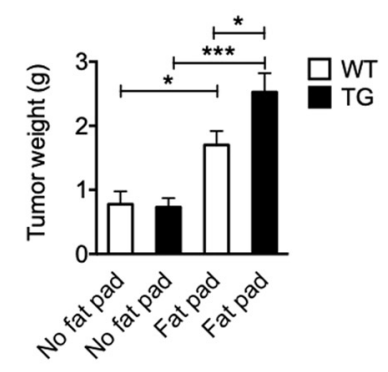

D

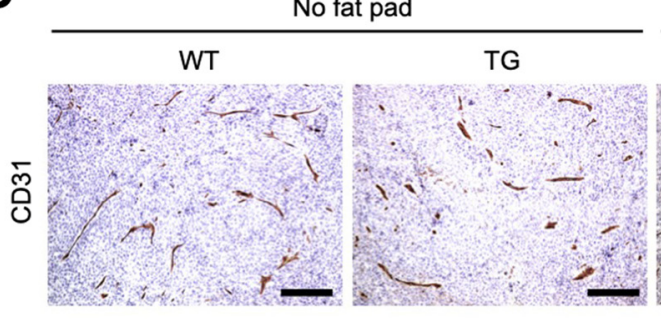

Fat pad

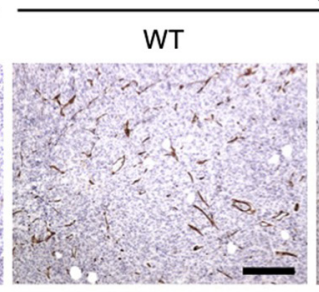

TG

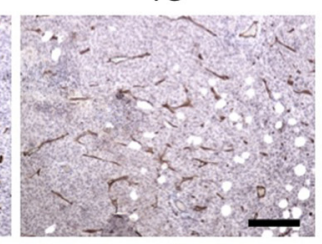

E

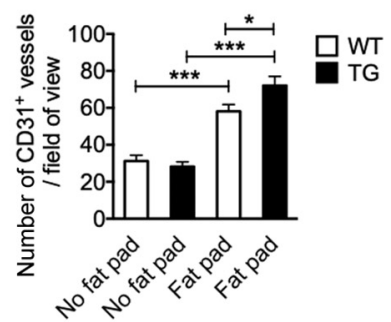

Figure 6 Blockade of lymphangiogenesis accelerates the growth of tumors implanted in the fat pad by affecting their vascularization. A: Graphical representation of the study design. B16F10 melanoma cells $\left(1 \times 10^{6}\right.$ cells) were implanted either in the fat pad or dorsal dermis (no fat pad) of wild-type (WT) or transgenic (TG) mice. B: Tumor growth profiles in WT and TG mice. C: Quantification of tumor weights. D: Immunohistochemical analysis of tumors implanted either in the fat pad or dorsal dermis (no fat pad) of WT or TG mice using an antibody against the CD31, the pan-endothelial cell marker. E: Blood vessel density quantification. Data are expressed as means \pm SEM. $n=6$ mice per group (B and $\mathbf{C}) ; n=3$ mice per group $(\mathbf{E})$. ${ }^{*} P<0.05,{ }^{* * *} P<0.001$ (statistical analysis with one-way analysis of variance followed by Tukey's multiple comparisons test). Scale bar $=200 \mu \mathrm{m}$ (D).

tumor-associated AT as previously observed. ${ }^{16}$ For that reason, B16F10 melanomas $\left(2.5 \times 10^{5}\right.$ cells $)$ alone or together with freshly isolated $\mathrm{F} 4 / 80^{+} \mathrm{CD} 11 \mathrm{~b}^{+}$AT macrophages $\left(2.5 \times 10^{5}\right.$ cells $)$ were implanted in contralateral fat pads of K14-VEGFR3-Ig mice (Figure 7A). Indeed, the presence of AT macrophages resulted in an accelerated growth of B16F10 melanomas (Figure 7B) characterized by an increased number of macrophages (Figure 7, C and D) and blood vessel density (Figure 7, E and F) as revealed by immunohistochemical analysis.

Because AT strongly accelerates mouse melanoma growth, OncoLnc was used to conduct overall survival analysis for $F A B P 4$, which encoded the fatty acid binding protein found in adipocytes, in 458 human skin cutaneous melanoma samples from TCGA database. The high expression of FABP4 was associated with poor overall survival $(P<0.0362)$ in cutaneous melanoma patients (Figure $8 \mathrm{~A}$ ). Spurred by this observation, it was determined whether correlation could be found between the expression levels of FABP4 with genes associated with macrophage infiltration after an analysis of 470 human skin cutaneous melanoma samples from TCGA database. First, a strong correlation was found among the gene expression levels of FABP4 and perilipin-1 (PLIN1). Of importance, the gene expression levels of $F A B P 4$ also correlated with IL6 as well as a marker of alternative macrophage polarization CLEC10A but not a pan-macrophage marker $C D 68$ (Figure 8B). We therefore hypothesized that the expression of IL- 6 might contribute to the presence of alternatively activated macrophages within tumor-associated AT in human melanomas. Indeed, the gene expression levels of IL6 strongly correlated with both CLECIOA and ILAR . Conversely, no correlation was found between IL6 and NOS2 expressed by classically activated macrophages (Figure $8 \mathrm{C}$ ). Instead, a strong correlation was observed between FABP4 and FLT4/VEGFR3 and lymphangiogenic growth factors such as $V E G F C$ and, to a lower extent, $V E G F D$. In addition, a correlation was observed between FLT4 and NOS2, implying a possible relationship between the presence of lymphatic vessels and classically activated macrophages (Figure 8D).

\section{Discussion}

To the best of our knowledge this is the first experimental study to address the role of lymphangiogenesis in the regulation of inflammatory response within tumorassociated AT. Here, we show that the blockade of VEGFR-3 activation renders AT primed or permissive for tumor growth through the accumulation of tumor-promoting macrophages.

Although intimately associated with the microenvironment of many tumors, AT is perhaps the most frequently overlooked stromal compartment. ${ }^{17,29,30}$ Nevertheless, it has recently been reported that AT is not a passive bystander in tumorigenesis. For example, tumor cells can influence adjacent adipocytes to acquire an activated phenotype 
A

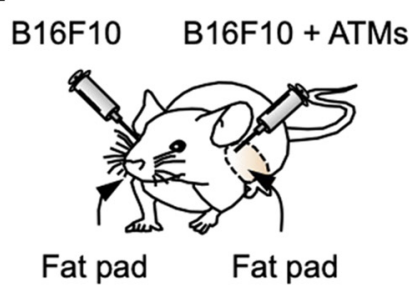

B

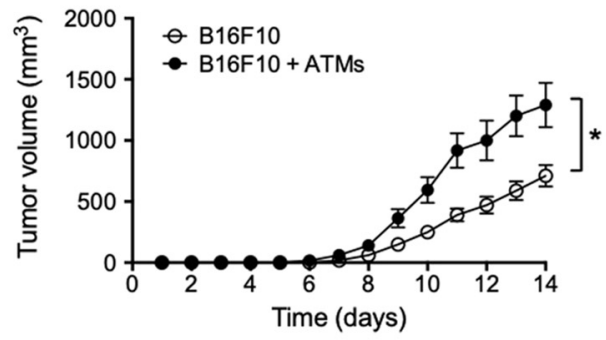

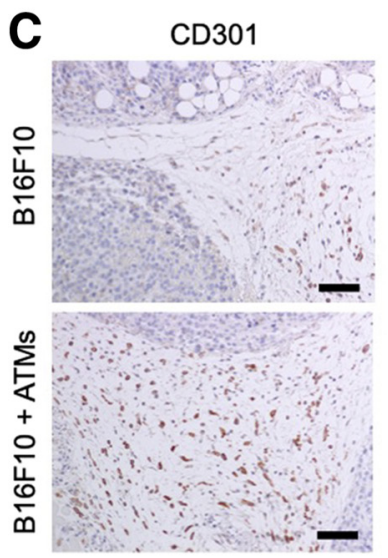
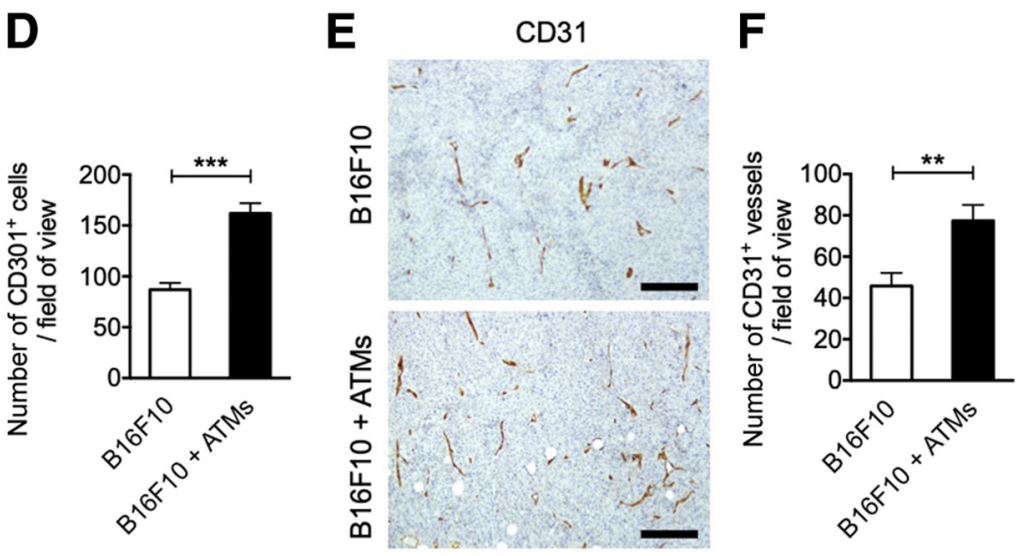

Figure 7 Adipose tissue macrophages (ATMs) stimulate the growth of tumors implanted in the fat pad. A: Graphical representation of the study design. B16F10 melanoma cells $\left(2.5 \times 10^{5}\right.$ cells $)$ without or with freshly isolated CD11b ${ }^{+}$F $/ 80^{+}$ATMs $\left(2.5 \times 10^{5}\right.$ cells $)$ were implanted in the fat pad of transgenic (TG) mice. Tumors were harvested 14 days after implantation. B: Growth profiles of tumors implanted without or with ATMs in the fat pad of TG mice. C and D: Immunohistochemical analysis (C) and quantification (D) of $\mathrm{CD}_{0} 1^{+}$cells in peritumoral AT from tumors implanted without or with ATMs in the fat pad of TG mice. $\mathbf{E}$ and $\mathbf{F}$ : Immunohistochemical analysis (E) and quantification (F) of $C D 31^{+}$vessels in peritumoral AT from tumors implanted without or with ATMs in the fat pad of TG mice. Data are expressed as means \pm SEM. $n=6$ mice per group $(\mathbf{A}$ and $\mathbf{B}) ; n=3$ mice per group $(\mathbf{C}-\mathbf{F}) ;{ }^{*} P<0.05,{ }^{*} P<0.01$, and $* * * P<0.001$ (statistical analysis with $t$-test). Scale bars: $50 \mu \mathrm{m}$ (C); $200 \mu \mathrm{m}$ (E).

characterized by reduced size and sustained lipolysis. ${ }^{31}$ In turn, tumor-associated adipocytes can provide a source of free fatty acids. ${ }^{31}$ The $\beta$-oxidation of free fatty acids can, therefore, fulfill the energy requirements of rapidly proliferating malignant cells. ${ }^{7,31}$ Adipocyte lipolysis might also contribute, at least in part, to an accelerated growth of tumors implanted in the fat pad of K14-VEGFR3-Ig mice, because the size of tumor-associated adipocytes was strikingly reduced. This phenotype was confirmed with the use of another model of lymphatic vessel dysfunction, CHY mice. Of note, the size of adipocytes from control AT decreased in K14-VEGFR3-Ig mice compared with adipocytes from WT counterparts. In CHY mice, however, the size of adipocytes from control AT increased relative to adipocytes from WT mice. This finding is consistent with the previous observation that the skin from CHY mice is characterized by an increased lipid accumulation compared with WT counterparts. ${ }^{32}$ Nevertheless, lipid droplets released from tumor-associated adipocytes in both K14-VEGFR3-Ig and CHY mice might promote macrophage recruitment and might augment an inflammatory response, resolution of which might depend on the lymphatic drainage function. ${ }^{33}$

Apart from lipid storage function, AT secretes cytokines such as IL-6. ${ }^{7}$ IL-6 is frequently viewed as a proinflammatory cytokine owing to its abundance in inflammatory settings such as obesity. ${ }^{34}$ However, accumulating evidence suggests a broader role for IL-6, including association with the inflammatory response resolution. Recently, it has been found that IL-6 stimulates proliferation as well as primes for IL4-dependent alternative activation of AT macrophages (ATMs) by inducing expression of IL-4R..$^{25-27}$ Alternatively activated macrophages have been implicated in the suppression of host antitumor immunity, stimulation of angiogenesis, and metastatic dissemination, contrarily to tumor-suppressive classically activated macrophages. ${ }^{35,36}$ It should be noted, however, that owing to their plastic potential the functional polarization of macrophages into only two groups is an oversimplification. Nevertheless, the local proliferation of ATMs could account for enrichment of macrophages expressing CD301/CLEC10A, a marker of alternative activation, in peritumoral AT from K14-VEGFR3-Ig mice. It has also been revealed that the exposure to T helper type 1 cytokines, such as tumor necrosis factor $\alpha$, inhibits the proliferation of ATMs. ${ }^{27}$ The development of tumor-associated inflammation and immunity relies on lymphatic vessels and their drainage function. ${ }^{4}$ Tumors implanted in K14VEGFR3-Ig mice exhibited drastically reduced expression of proinflammatory mediators compared with those implanted in WT littermates. ${ }^{4}$ Accounting for this feature, 
A

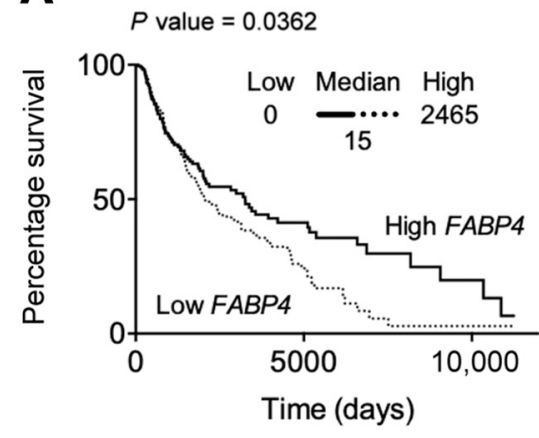

B

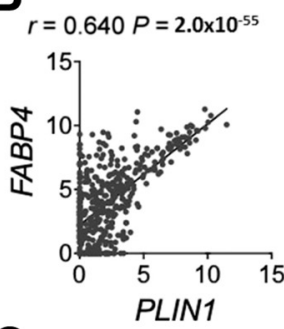

C
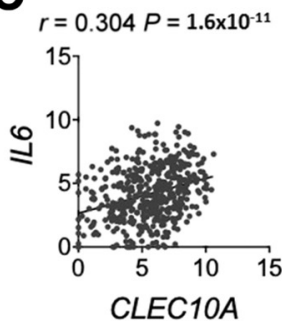

D

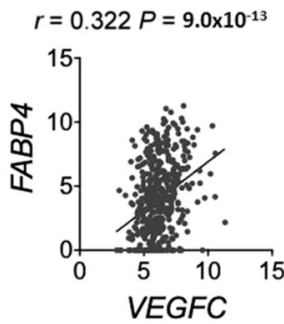

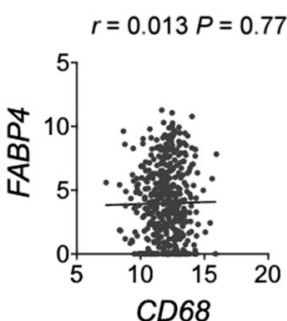
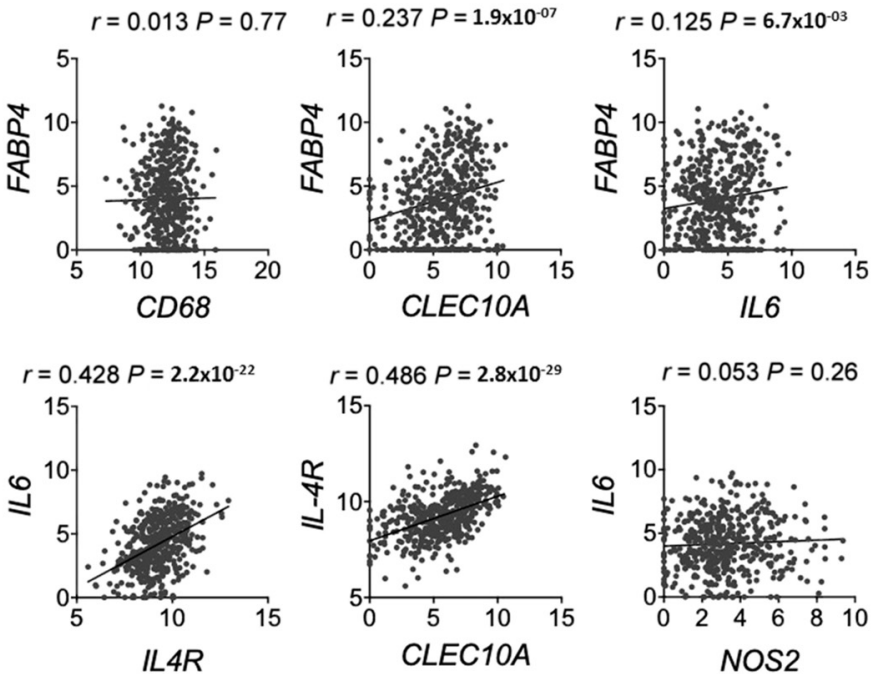

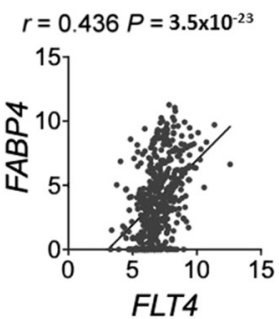

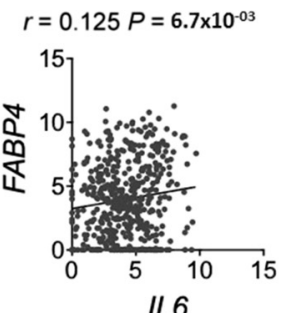

IL6

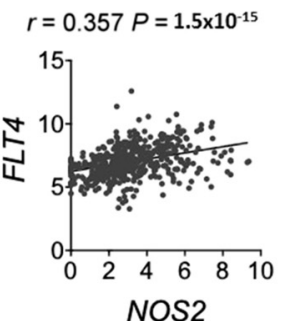

Figure 8 The expression of adipocyte marker FABP4 is associated with poor overall survival in cutaneous melanoma patients. A: Overall survival of skin cutaneous melanoma (SKCM) patients generated using OncoLnc platform. The Cancer Genome Atlas (TCGA) data of 458 melanoma patients were assigned into low or high groups according to the expression level of FABP4 reported as RNASeq values. Patients displaying an FABP4 expression level ranging from 0 to 15 were considered as low (solid curve) and patients displaying FABP4 expression level ranging from 15 to 2465 were considered as high (dotted curve). B-D: Correlation of gene expression levels using data set acquired from R2 platform containing 470 cutaneous melanoma patients (Tumor Skin Cutaneous Melanoma - TCGA - 470 - rsem - tcgars). Black lines denote the linear regression. Pearson's correlation coefficient, $r$, is shown.

macrophages from tumor-associated AT from K14VEGFR3-Ig mice might be exposed to the microenvironment facilitating their proliferation.

The importance of ATMs in facilitating tumor growth was demonstrated by the fact that melanomas coimplanted with freshly isolated macrophages grew more rapidly and were significantly more vascularized than those implanted alone. The extent to which ATMs contribute to the disease progression in humans remains to be deciphered. With the use of a cohort of 458 cutaneous skin melanoma patients, it was demonstrated that the expression of FABP4 is associated with poor overall survival. Of importance, expression of FABP4 correlated positively with CLEC1OA. It is therefore tempting to speculate whether the presence of macrophages within tumor-associated AT in human melanomas contributes to the disease progression.

It is now recognized that macrophages express angiogenic factors, including VEGF-A and IL- $6 .{ }^{37}$ Of interest, it was revealed that IL- 6 can directly induce angiogenesis in a variety of models with similar potency to VEGF-A. ${ }^{38}$ This finding together with increased levels of IL-6 observed in peritumoral AT from K14-VEGFR3-Ig mice suggest that IL-6 might play an important proangiogenic role in our model. The extent to which adipocytes and macrophages separately contribute to this process remains to be assessed.

In several solid tumor types, VEGFR-3 has been found expressed on angiogenic blood vessels. ${ }^{39}$ In addition, angiogenic sprouting and vascular network formation have been inhibited by blockade of the receptor. ${ }^{40}$ However, the expression of VEGFR-3 has been confided primarily to the vasculature within the tumor tissue. Because tumors expand at their periphery, peritumoral AT might therefore serve as a source of new blood vessels. Indeed, apart from sprouting angiogenesis, several other mechanisms of neovascularization have been identified in solid tumors, including vessel co-option. ${ }^{41} \mathrm{We}$ might only speculate whether this process accounts for an increased number of $\mathrm{CD} 31^{+}$structures within tumors implanted into the fat pad of K14-VEGFR3-Ig mice.

Taken together, these results identify a complex role of lymphangiogenesis in tumor progression that requires further investigation. Tumor-associated lymphangiogenesis contributes to malignant progression, at least in part, by 
facilitating metastatic spread to regional lymph nodes. These data suggest that the blockade of VEGFR-3 signaling aids in the induction of an inflammatory response within AT associated with accumulation of alternatively activated macrophages that stimulate tumor growth and vascularization. The development of an inducible mouse model with a spatiotemporal regulation of lymphatic vessels in and around the tumor tissue may prove useful in dissecting the dynamics of an inflammatory response. The contribution of lymphatic vessels to the local inflammatory response within tumor-associated AT may be of therapeutic importance for tumors growing within AT or metastases to the lymph nodes.

\section{Acknowledgments}

The $\mathrm{C} 3 \mathrm{H} 101 \mathrm{H}-\mathrm{Flt} 4<\mathrm{Chy}>/ \mathrm{H}$ mice (repository number: EM:00068) were obtained from the MRC-Harwell on behalf of the European Mouse Mutant Archive. K14-VEGFR3-Ig male mice were a gift from Prof. Kari Alitalo (University of Helsinki, Finland).

\section{References}

1. Wiig H, Swartz MA: Interstitial fluid and lymph formation and transport: physiological regulation and roles in inflammation and cancer. Physiol Rev 2012, 92:1005-1060

2. Karaman S, Detmar M: Mechanisms of lymphatic metastasis. J Clin Invest 2014, 124:922-928

3. Kimura T, Sugaya M, Oka T, Blauvelt A, Okochi H, Sato S: Lymphatic dysfunction attenuates tumor immunity through impaired antigen presentation. Oncotarget 2015, 6:18081-18093

4. Lund AW, Wagner M, Fankhauser M, Steinskog ES, Broggi MA, Spranger S, Gajewski TF, Alitalo K, Eikesdal HP, Wiig H, Swartz MA: Lymphatic vessels regulate immune microenvironments in human and murine melanoma. J Clin Invest 2016, 126:3389-3402

5. Steinskog ES, Sagstad SJ, Wagner M, Karlsen TV, Yang N, Markhus CE, Yndestad S, Wiig H, Eikesdal HP: Impaired lymphatic function accelerates cancer growth. Oncotarget 2016, 7:45789-45802

6. Escobedo N, Oliver G: The lymphatic vasculature: its role in adipose metabolism and obesity. Cell Metab 2017, 26:598-609

7. Wagner M, Dudley AC: A three-party alliance in solid tumors: adipocytes, macrophages and vascular endothelial cells. Adipocyte 2013, 2:67-73

8. Weisberg SP, McCann D, Desai M, Rosenbaum M, Leibel RL, Ferrante AW Jr: Obesity is associated with macrophage accumulation in adipose tissue. J Clin Invest 2003, 112:1796-1808

9. Hotamisligil GS, Shargill NS, Spiegelman BM: Adipose expression of tumor necrosis factor-alpha: direct role in obesity-linked insulin resistance. Science 1993, 259:87-91

10. Uysal KT, Wiesbrock SM, Marino MW, Hotamisligil GS: Protection from obesity-induced insulin resistance in mice lacking TNF-alpha function. Nature 1997, 389:610-614

11. Lumeng CN, Bodzin JL, Saltiel AR: Obesity induces a phenotypic switch in adipose tissue macrophage polarization. J Clin Invest 2007, 117:175-184

12. Sartipy P, Loskutoff DJ: Monocyte chemoattractant protein 1 in obesity and insulin resistance. Proc Natl Acad Sci U S A 2003, 100:7265-7270

13. Wagner M, Steinskog ESS, Wiig H: Adipose tissue macrophages: the inflammatory link between obesity and cancer? Expert Opin Ther Targets 2015, 19:527-538
14. Wagner M: A dangerous duo in adipose tissue: high-mobility group box 1 protein and macrophages. Yale J Biol Med 2014, 87:127-133

15. Karaman S, Hollmen M, Robciuc MR, Alitalo A, Nurmi H, Morf B, Buschle D, Alkan HF, Ochsenbein AM, Alitalo K, Wolfrum C, Detmar M: Blockade of VEGF-C and VEGF-D modulates adipose tissue inflammation and improves metabolic parameters under highfat diet. Mol Metab 2015, 4:93-105

16. Wagner M, Bjerkvig R, Wiig H, Melero-Martin JM, Lin RZ, Klagsbrun M, Dudley AC: Inflamed tumor-associated adipose tissue is a depot for macrophages that stimulate tumor growth and angiogenesis. Angiogenesis 2012, 15:481-495

17. Wagner M, Bjerkvig R, Wiig H, Dudley AC: Loss of adipocyte specification and necrosis augment tumor-associated inflammation. Adipocyte 2013, 2:176-183

18. Makinen T, Jussila L, Veikkola T, Karpanen T, Kettunen MI, Pulkkanen KJ, Kauppinen R, Jackson DG, Kubo H, Nishikawa S, Yla-Herttuala S, Alitalo K: Inhibition of lymphangiogenesis with resulting lymphedema in transgenic mice expressing soluble VEGF receptor-3. Nat Med 2001, 7:199-205

19. Irrthum A, Karkkainen MJ, Devriendt K, Alitalo K, Vikkula M: Congenital hereditary lymphedema caused by a mutation that inactivates VEGFR3 tyrosine kinase. Am J Hum Genet 2000, 67:295-301

20. Karkkainen MJ, Saaristo A, Jussila L, Karila KA, Lawrence EC, Pajusola K, Bueler H, Eichmann A, Kauppinen R, Kettunen MI, Yla-Herttuala S, Finegold DN, Ferrell RE, Alitalo K: A model for gene therapy of human hereditary lymphedema. Proc Natl Acad Sci U S A 2001, 98:12677-12682

21. Lim HY, Lim SY, Tan CK, Thiam CH, Goh CC, Carbajo D, Chew SHS, See P, Chakarov S, Wang XN, Lim LH, Johnson LA, Lum J, Fong CY, Bongso A, Biswas A, Goh C, Evrard M, Yeo KP, Basu R, Wang JK, Tan Y, Jain R, Tikoo S, Choong C, Weninger W, Poidinger M, Stanley RE, Collin M, Tan NS, Ng LG, Jackson DG, Ginhoux F, Angeli V: Hyaluronan receptor LYVE-1-expressing macrophages maintain arterial tone through hyaluronan-mediated regulation of smooth muscle cell collagen. Immunity 2018, 49: 326-341.e7

22. Cho CH, Koh YJ, Han J, Sung HK, Jong Lee H, Morisada T, Schwendener RA, Brekken RA, Kang G, Oike Y, Choi TS, Suda T, Yoo OJ, Koh GY: Angiogenic role of LYVE-1-positive macrophages in adipose tissue. Circ Res 2007, 100:e47-e57

23. Gordon EJ, Rao S, Pollard JW, Nutt SL, Lang RA, Harvey NL: Macrophages define dermal lymphatic vessel calibre during development by regulating lymphatic endothelial cell proliferation. Development 2010, 137:3899-3910

24. Harvey NL, Gordon EJ: Deciphering the roles of macrophages in developmental and inflammation stimulated lymphangiogenesis. Vasc Cell 2012, 4:15

25. Fernando MR, Reyes JL, Iannuzzi J, Leung G, McKay DM: The proinflammatory cytokine, interleukin-6, enhances the polarization of alternatively activated macrophages. PLoS One 2014, 9:e94188

26. Mauer J, Chaurasia B, Goldau J, Vogt MC, Ruud J, Nguyen KD, Theurich S, Hausen AC, Schmitz J, Bronneke HS, Estevez E, Allen TL, Mesaros A, Partridge L, Febbraio MA, Chawla A, Wunderlich FT, Bruning JC: Signaling by IL-6 promotes alternative activation of macrophages to limit endotoxemia and obesityassociated resistance to insulin. Nat Immunol 2014, 15:423-430

27. Braune J, Weyer U, Hobusch C, Mauer J, Bruning JC, Bechmann I, Gericke M: IL-6 regulates M2 polarization and local proliferation of adipose tissue macrophages in obesity. J Immunol 2017, 198: $2927-2934$

28. Cho KW, Morris DL, Lumeng CN: Flow cytometry analyses of adipose tissue macrophages. Methods Enzymol 2014, 537: $297-314$

29. Fearon KC, Glass DJ, Guttridge DC: Cancer cachexia: mediators, signaling, and metabolic pathways. Cell Metab 2012, 16:153-166

30. Baracos VE, Martin L, Korc M, Guttridge DC, Fearon KCH: Cancerassociated cachexia. Nat Rev Dis Primers 2018, 4:17105 
31. Zhang M, Di Martino JS, Bowman RL, Campbell NR, Baksh SC, Simon-Vermot T, Kim IS, Haldeman P, Mondal C, YongGonzales V, Abu-Akeel M, Merghoub T, Jones DR, Zhu XG, Arora A, Ariyan CE, Birsoy K, Wolchok JD, Panageas KS, Hollmann T, Bravo-Cordero JJ, White RM: Adipocyte-derived lipids mediate melanoma progression via FATP proteins. Cancer Discov 2018, 8:1006-1025

32. Rutkowski JM, Markhus CE, Gyenge CC, Alitalo K, Wiig H, Swartz MA: Dermal collagen and lipid deposition correlate with tissue swelling and hydraulic conductivity in murine primary lymphedema. Am J Pathol 2010, 176:1122-1129

33. Sugimoto MA, Vago JP, Perretti M, Teixeira MM: Mediators of the resolution of the inflammatory response. Trends Immunol 2019, 40: 212-227

34. Mauer J, Denson JL, Bruning JC: Versatile functions for IL-6 in metabolism and cancer. Trends Immunol 2015, 36:92-101

35. Mantovani A, Locati M: Tumor-associated macrophages as a paradigm of macrophage plasticity, diversity, and polarization: lessons and open questions. Arterioscler Thromb Vasc Biol 2013, 33: $1478-1483$

36. Condeelis J, Pollard JW: Macrophages: obligate partners for tumor cell migration, invasion, and metastasis. Cell 2006, 124: $263-266$
37. Corliss BA, Azimi MS, Munson JM, Peirce SM, Murfee WL: Macrophages: an inflammatory link between angiogenesis and lymphangiogenesis. Microcirculation 2016, 23:95-121

38. Gopinathan G, Milagre C, Pearce OM, Reynolds LE, HodivalaDilke K, Leinster DA, Zhong H, Hollingsworth RE, Thompson R, Whiteford JR, Balkwill F: Interleukin-6 stimulates defective angiogenesis. Cancer Res 2015, 75:3098-3107

39. Smith NR, Baker D, James NH, Ratcliffe K, Jenkins M, Ashton SE, Sproat G, Swann R, Gray N, Ryan A, Jurgensmeier JM, Womack C: Vascular endothelial growth factor receptors VEGFR-2 and VEGFR-3 are localized primarily to the vasculature in human primary solid cancers. Clin Cancer Res 2010, 16:3548-3561

40. Tammela T, Zarkada G, Wallgard E, Murtomaki A, Suchting S, Wirzenius M, Waltari M, Hellstrom M, Schomber T, Peltonen R, Freitas C, Duarte A, Isoniemi H, Laakkonen P, Christofori G, YlaHerttuala S, Shibuya M, Pytowski B, Eichmann A, Betsholtz C, Alitalo K: Blocking VEGFR-3 suppresses angiogenic sprouting and vascular network formation. Nature 2008, 454:656-660

41. Donnem T, Hu J, Ferguson M, Adighibe O, Snell C, Harris AL, Gatter KC, Pezzella F: Vessel co-option in primary human tumors and metastases: an obstacle to effective anti-angiogenic treatment? Cancer Med 2013, 2:427-436 